\title{
Dynamics Modeling of Harmonic Reducer and Analysis of Flexspline Fatigue
}

\author{
GongXue Zhang ${ }^{1, a}$ and Che Ma ${ }^{2, b, *}$ \\ ${ }^{1}$ Shaanxi University of Science \& Technology,Xi'an, China \\ ${ }^{2}$ Shaanxi University of Science \& Technology,Xi'an, China \\ a448344839@qq.com, bmache01@163.com \\ ${ }^{*}$ Che Ma
}

Keywords: Harmonic reducer, Dynamic model, Flexspline, Fatigue failure.

\begin{abstract}
The paper takes the static and dynamic error of the harmonic gear drive into a full account, bases on the harmonic drive model to establish a system dynamics model and simplifies the infinite degree of freedom of harmonic gear driving system as a multi-degree-of-freedom system to derive the dynamic response to the stimulus under the physical coordinates. At the same time, the transfer function matrix and the frequency response function matrix after was obtained based on the multi-degree-of-freedom system. What is more, ADAMS software was used to establish a holistic rigid-flexible coupling model whose verification was done and the ANSYS Workbench software was used to judge the position where the flexspline is prone to suffer from fatigue by means of the transient dynamic model.
\end{abstract}

\section{Introduction}

Harmonic Reducer is the use of its components of the wave transmission and motion conversion principle to achieve deceleration movement, which has the advantages that general transmission can't match. Due to the existence of flexible component with controllable deformation, the research of the problem becomes more complicated[1]. Therefore, many scholars focus on the study of the flexspline. The flexspline produces forced deformation under the oppression of the wave generator, which makes the flexspline produces fatigue damage easily, resulting in reduced performance of the machine. The dynamic behavior and modeling methods of harmonic drive system were reviewed by Ma donghui. He pointed out that the lack of dynamic model[2]. A full research between the rotational speed and meshing force of software simulation with theoretical value wear made by Shi guangzu[3]. For the mechanics and fatigue analysis of the flexspline, many scholars have simplified the model and obtained many valuable results[4,5]. The paper based on the establishment of the system dynamics model, the transient dynamic model is used to verify and obtain the position of the fatigue failure of the flexspline.

\section{Dynamic Model}

Flexspline and wave generator of harmonic reducer forced deformation, with the staggered tooth phenomenon of the external gear of the flexspline and the internal gear of the rigid circular spline in meshing, making the overall flex-rigid multibody system becomes more complex. Taking the static and dynamic error of the harmonic gear drive into a full account, combined with the non-linear of torsional stiffness, the paper establishes the dynamic mechanical model of harmonic gear transmission system. The physical model is shown in figure 1. The kinetic energy of the system is mainly determined by the input high speed of the harmonic reducer and the low output speed. The kinetic energy formula is:

$$
T=\frac{1}{2} J_{m} \dot{\theta}_{m}^{2}+\frac{1}{2} J_{I} \dot{\theta}_{l}^{2}
$$


Which, $J_{m}$ is the input moment of inertia, $J_{1}$ is the output moment of inertia, $\theta_{m}$ is the generalized independent coordinate system of input shaft, $\theta_{l}$ is the generalized independent coordinate system of output shaft.

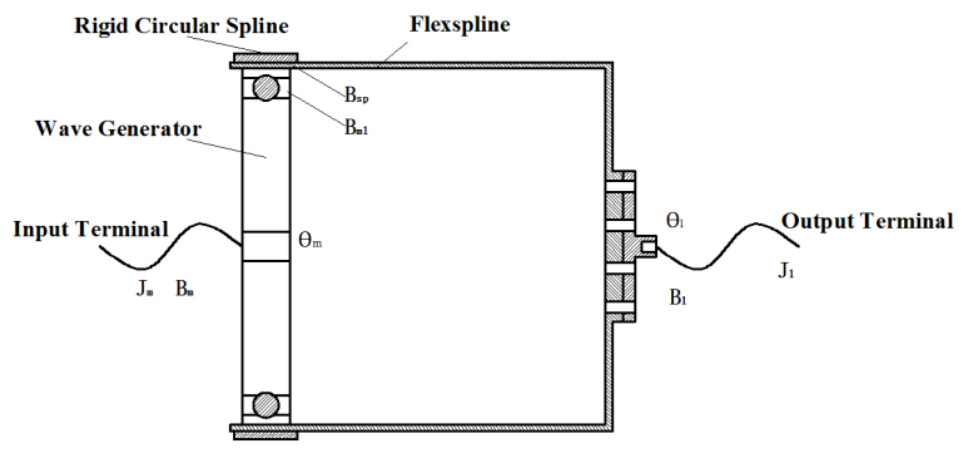

Fig.1 Physical model of harmonic reducer. we have established physical model, which is Moment of inertia and comprehensive damping of input terminal and output terminal.

According to the error source analysis and the physical model of harmonic drive, the transmission model shown in figure 2 is established to set the dynamic equation.

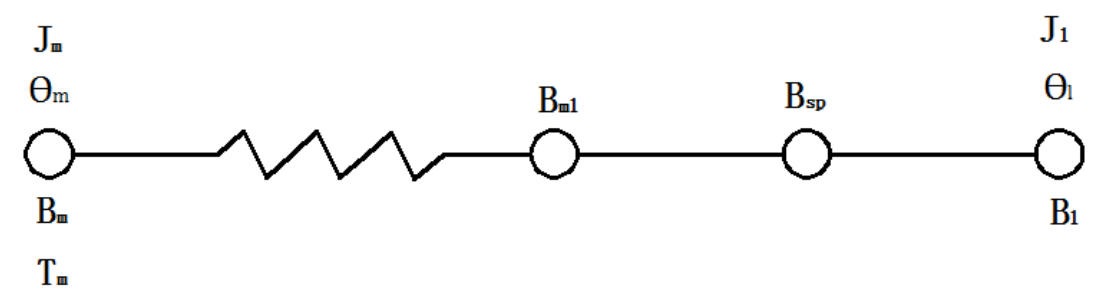

Fig. 2 transmission model of harmonic reducer. The harmonic drive model is established.

the system potential is:

$$
V=\int_{0}^{\theta_{1}-\theta_{m} / N+\tilde{\theta}_{p}} K(\varphi) \varphi d \varphi
$$

Rayleigh's dissipation function of system is:

$$
D=\frac{1}{2} B_{m} \dot{\theta}_{m}^{2}+\frac{1}{2} B_{l} \dot{\theta}_{l}^{2}+\frac{1}{2} B_{m l}\left(\dot{\theta}_{m}+\dot{\theta}_{l}\right)^{2}+\frac{1}{2} B_{s p}\left(\dot{\theta}_{l}-\frac{\dot{\theta}_{m}}{N}+\tilde{\theta}_{p}\right)^{2}
$$

Therefore, the Lagrange equation for harmonic drive is:

$$
\begin{gathered}
T_{m}=\frac{d}{d t}\left(\frac{\partial T}{\partial \dot{\theta}_{m}}\right)-\frac{\partial T}{\partial \theta_{m}}+\frac{\partial V}{\theta_{m}}+\frac{\partial D}{\partial \dot{\theta}_{m}} \\
0=\frac{d}{d t}\left(\frac{\partial T}{\partial \dot{\theta}_{l}}\right)-\frac{\partial T}{\partial \theta_{l}}+\frac{\partial V}{\theta_{1}}+\frac{\partial D}{\partial \dot{\theta}_{l}}
\end{gathered}
$$

The equation can be written as:

$$
\begin{gathered}
T_{m}=J_{m} \ddot{\theta}_{m}+\left[k_{1}+k_{2}\left(\theta_{l}-\frac{\theta_{m}}{N}+\tilde{\theta}_{p}\right)^{2}\right]\left(\frac{d \tilde{\theta}_{p}}{d \theta_{m}}-\frac{1}{N}\right) \\
+B_{m l}\left(\dot{\theta}_{m}+\dot{\theta}_{l}\right)+B_{m} \dot{\theta}_{m}+B_{s p}\left(\frac{d \tilde{\theta}_{p}}{d \theta_{m}}-\frac{1}{N}\right)\left(\dot{\theta}_{l}+\dot{\tilde{\theta}}_{p}-\frac{\dot{\theta}_{m}}{N}\right) \\
0=J_{l} \ddot{\theta}_{l}+\left[k_{1}+k_{2}\left(\theta_{l}+\tilde{\theta}_{p}-\frac{\theta_{m}}{N}\right)^{2}\right]+B_{m l}\left(\dot{\theta}_{m}+\dot{\theta}_{l}\right)+B_{l} \dot{\theta}_{l}+B_{s p}\left(\dot{\theta}_{l}+\dot{\tilde{\theta}}_{p}-\frac{\dot{\theta}_{m}}{N}\right)
\end{gathered}
$$

Which, $B_{m}$ is the comprehensive damping of input terminal, $B_{1}$ is the comprehensive damping of output terminal, $B_{m l}$ is the damping in the Flexspline, $B_{s p}$ is the damping in the rigid circular spline, $T_{m}$ is the input moment. 


\section{Dynamic Response}

The actual transmission structure analysis of the harmonic reducer is more complex, the actual structure with infinite free elastic body, it is not easy to precise handle system vibration, and in many cases, there is no need to deal with like that, so in order to combine with the actual, we simplify the complexity of the problem, which makes an infinite degree of freedom system to form a multi-degree-of-freedom system. Assuming that the system is not damped, the forced vibration equation of the $\mathrm{N}$-degree-of-freedom system is:

$$
P(t)=[M] \ddot{U}(t)+[K] U(t)
$$

Which, $P(t)$ is the random vector of exciting force. The mode superposition method is used to solve the problem of dynamic response. Assuming that $\psi_{i}$ is the $\mathrm{i}$-order regular mode, obtaining the regular mode matrix $[\psi],[\psi]=\left[\psi_{1}, \psi_{2}, \cdots, \psi_{n}\right]$, then $[\psi]$ as the transformation matrix, transformed into $U(t)=[\psi] \xi(t), \xi(t)$ is the regular system coordinates, then the normal vibration equation under the regular coordinates:

$$
[\psi]^{T}[M][\psi] \ddot{\xi}(t)+[\psi]^{T}[K][\psi] \xi(t)=[\psi]^{T} P(t)
$$

This can also be written as:

$$
[I] \ddot{\xi}(t)+[\mathrm{X}] \xi(t)=R(t)
$$

Which, $[I]$ is unit matrix, $[\mathrm{X}]$ is the characteristic matrix, at this point,

$$
[\mathrm{X}]=\left[\begin{array}{cccc}
\omega_{1}^{2} & 0 & 0 & 0 \\
0 & \omega_{2}^{2} & 0 & 0 \\
0 & 0 & \cdots & 0 \\
0 & 0 & 0 & \omega_{n}^{2}
\end{array}\right]
$$

$R(t)$ in equation is exciting force in canonical coordinates, written as:

$$
R(t)=\left[\begin{array}{c}
R_{1}(t) \\
R_{2}(t) \\
\cdots \\
R_{3}(t)
\end{array}\right]=[\psi]^{T} P(t)\left[\begin{array}{c}
\psi_{1}^{T} P(t) \\
\psi_{2}^{T} P(t) \\
\cdots \\
\psi_{n}^{T} P(t)
\end{array}\right]
$$

Where the i-th equation is:

$$
\ddot{\xi}_{i}(t)+\omega_{i}^{2} \xi(t)=R_{i}(t)
$$

The response of the system to the incentive under physical coordinates is:

$$
U(t)=[\psi] \xi(t)=\left[\psi_{1}, \psi_{2}, \cdots, \psi_{n}\right]\left[\begin{array}{c}
\xi_{1}(t) \\
\xi_{2}(t) \\
\ldots \\
\xi_{n}(t)
\end{array}\right]=\sum_{i=1}^{n} \psi_{i} \xi_{i}(t)
$$

Harmonic reducer is a multi-degree of freedom system, its own degree of freedom system is more complex, when the external motor input a high speed to the harmonic reducer, the motion equation of the system is :

$$
[m]\{\ddot{x}\}+[c]\{\dot{x}\}+[k]\{x\}=\{f(t)\}
$$

For its Laplace transformation can be obtained:

$$
\left(s^{2}[m]+s[c]+[k]\right)\{X(s)\}=\{F(s)\}
$$

Then, the transfer function can be expressed as:

$$
[H(s)]=\frac{\{X(s)\}}{\{F(s)\}}=\frac{1}{s^{2}[m]+s[c]+[k]}
$$

This is a transfer function of the N-order equation. When $s=j \omega$, the frequency response function matrix can be expressed as: 


$$
[H(s)]_{s=j \omega}=[H(\omega)]=\frac{\{X(\omega)\}}{\{F(\omega)\}}=\frac{1}{j \omega[c]+[k]-\omega^{2}[m]}
$$

Expand definition of the transfer function, it is $\{X(s)\}=[H(s)]\{F(s)\}$, then expressed as:

$$
\left\{\begin{array}{c}
X_{1}(s) \\
X_{2}(s) \\
\cdots \\
X_{N}(s)
\end{array}\right\}=\left[\begin{array}{cccc}
H_{11}(s) & H_{12}(s) & \cdots & H_{1 N}(s) \\
H_{21}(s) & H_{22}(s) & \cdots & H_{2 N}(s) \\
\cdots & \cdots & \ddots & \cdots \\
H_{N 1}(s) & H_{N 2}(s) & \cdots & H_{N N}(s)
\end{array}\right]\left\{\begin{array}{c}
F_{1}(s) \\
F_{2}(s) \\
\cdots \\
F_{N}(s)
\end{array}\right\}=\left[\begin{array}{l}
\sum_{k=1}^{N} H_{1 k}(s) F_{k}(s) \\
\sum_{k=1}^{N} H_{2 k}(s) F_{k}(s) \\
\cdots \\
\sum_{k=1}^{N} H_{N k}(s) F_{k}(s)
\end{array}\right]
$$

For any coordinates, the Laplace transformation of response $x_{e}(t)$ is:

$$
x_{e}(s)=\sum_{k=1}^{N} H_{e k}(s) F_{k}(s)
$$

It represents the Laplace transform of the e-th physical coordinate displacement response of the system, it is equal to the product algebraic sum of the Laplace transformations of the forces and other transfer functions. With the basis of the transfer function matrix and the frequency response function matrix, we can carry out the next step of the modal test.

\section{Dynamic Simulation Analysis}

Firstly, the rigid-flexible coupling model is established in ADAMS software, and the displacement-time curve is obtained by virtual prototyping, to verify the correctness of the modeling. The time-displacement curves of the Flexspline and the virtual prototype are shown in figure 3 and figure 4.

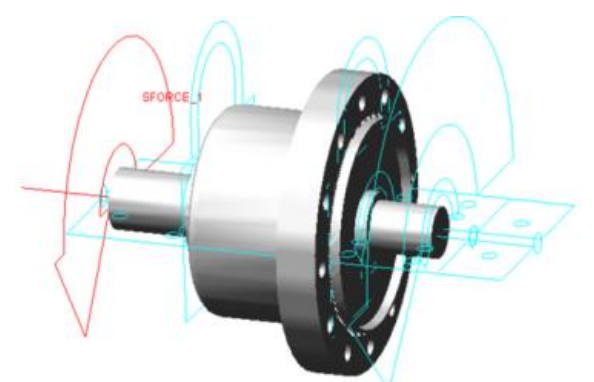

Fig.3 Virtual prototype of harmonic reducer. A virtual prototype of constraints and loads is added.

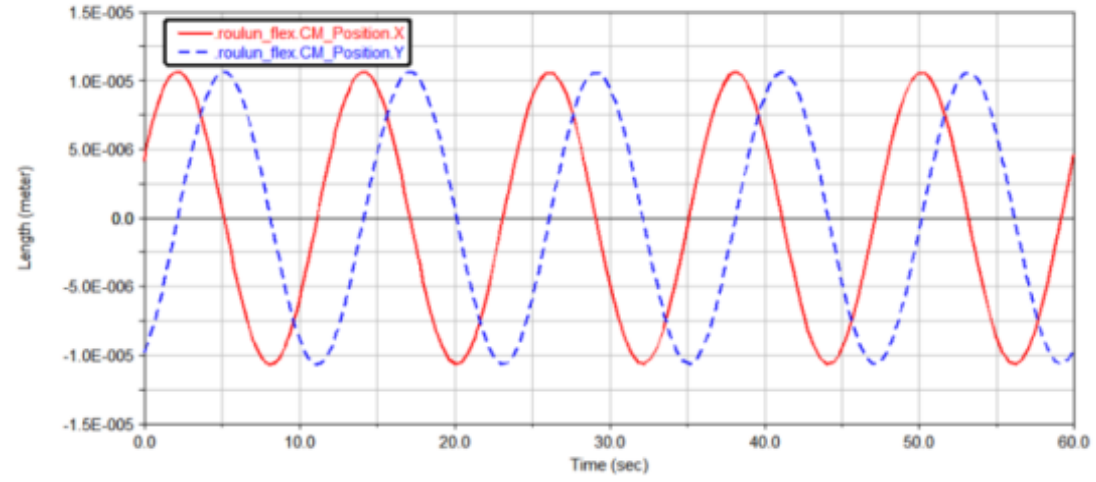

Fig.4 Displacement in $\mathrm{x}$ and y-direction - time curve of flexspline. The curve of $\mathrm{x}$ and the curve of $\mathrm{y}$ showed a simple harmonic fluctuation.

In the model, the material of flexspline is $30 \mathrm{CrMnsi}$, the rigid circular spline and wave generator is 45 steel material. The material properties of each component are shown in Table 1. 
Tab.1 Material Properties of Each Part. Using different materials can improve the overall performance.

\begin{tabular}{|c|c|c|c|c|c|}
\hline Parts & Material & $\begin{array}{c}\text { Elastic } \\
\text { modulus/ } \mathrm{Gpa}\end{array}$ & Poisson's ratio / $\mu$ & Density/g/ $/ \mathrm{cm}^{3}$ & $\begin{array}{c}\text { Yield limit } \\
/ \mathrm{Mpa}\end{array}$ \\
\hline Flexspline & $30 \mathrm{CrMnSiA}$ & 204 & 0.29 & 6.691 & 885 \\
\hline Rigid Circular Spline & 45 & 210 & 0.3 & 7.85 & 355 \\
\hline Wave Generator & 45 & 210 & 0.3 & 7.85 & 355 \\
\hline
\end{tabular}

Dividing the free mesh of the flexspline, the results shown in Figure 5, where the number of nodes is 44623 , the number of units is 23435 .

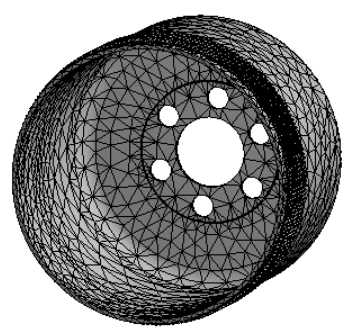

Fig.5 Meshing of flexspline.

Under different confinement states, the natural frequency and vibration mode of the structure will change, and the modal analysis after applying the constraint can reflect the real vibration of the structure. The first 8 order mode is analyzed, and the frequency and mode of the 4,6 , and 8 order flexspline are shown in figure 6.

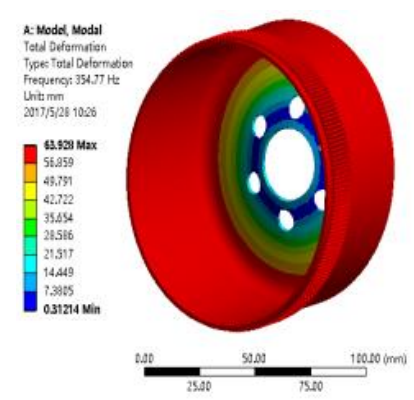

a) Inherent frequency and vibration mode of the fourth order. almost non-defrmation, The maximum deformation is $63.928 \mathrm{~mm}$.

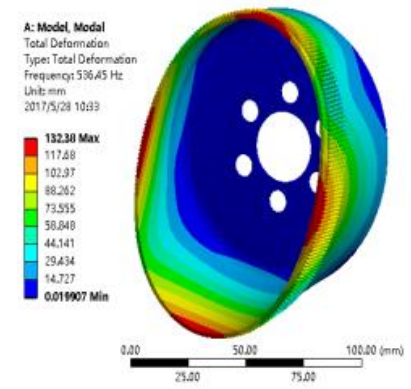

b) Inherent frequency and vibration mode of the sixth order. The cup of the flexspline presents elliptical deformation.

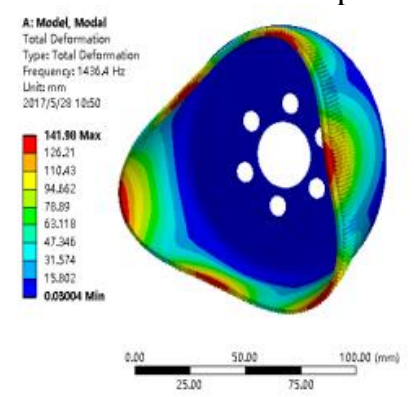

c) Inherent frequency and vibration mode of the eighth order. The shape of the cup of the flexspline is triangular.

Fig.6 Vibration chart of modes of the flexspline 
Through the transient dynamic analysis of the flexspline, the equivalent stress and equivalent strain distributions of the flexspline are obtained by solving the transient dynamic model, as shown in figure 7 and figure 8 .

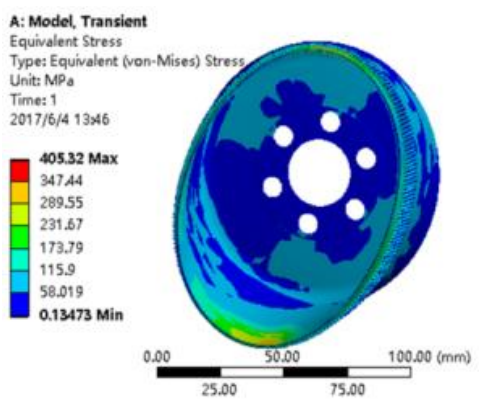

Fig.7 The distribution cloud map of equivalent stress of the flexspline. Stress concentration is observed at both ends of the cup.

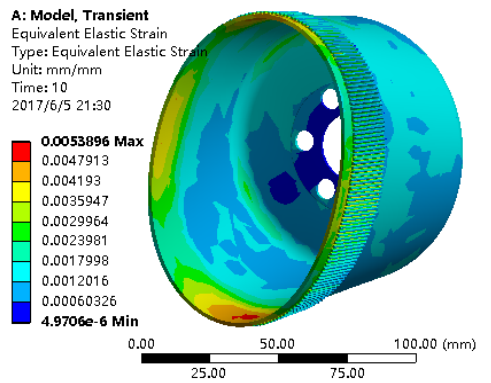

Fig.8 The cloud map of equivalent strain of the flexspline. This position is most vulnerable to fatigue damage.

From the distribution cloud map of equivalent stress and the cloud map of equivalent strain of the flexspline, it can be seen that the symmetrical position of the two sides of the vertebral cup deformation is prone to stress concentration, so this position is prone to fatigue damage.

\section{Ending}

(1) The system dynamics model is established by way of the harmonic drive physical model through the error source analysis so that the transfer function matrix and the frequency response function matrix could be obtained.(2) The holistic rigid-flexible coupling model was established. After the verification of the model, the transient dynamic model was used for a solution so as to find out that the symmetrical parts at two sides of the flexspline rim are prone to be exposed to fatigue.

\section{Acknowledgement}

This research was financially supported by the Technology Major Foundation of China (Grant NO.2009ZX04001-014) and the Natural Science Research Funds for Shaanxi (Grant NO.2014JM7264).

\section{References}

[1] W. Chang-Ming, Summary of status on the harmonic gear driving technology, Journal of mechanical transmission, vol.30, pp. 86-88, 2006.

[2] M. Dong-Hui, Advances in researches of dynamic models in harmonic drive system, China science paper, vol.10, pp. 1983-1990, 2015.

[3] S. Zu-Guang, Harmonic gear based recur dyn software model of multi-body dynamic analysis, Hunan agricultural machinery, vol.38, pp. 99-100, 2011.

[4] D. Juan, Stress analysis and parameter optimization of column-shaped flexspline in harmonic drive based on ansys, Machinery design and manufacture, vol.04, pp. 59-61, 2012.

[5] W. Yan-Feng, Finite element mechanics analysis of flexspline in harmonic driver and improvement of structural parameter, Optics and precision engineering, vol.11, pp. 86-90, 2005. 
[6] Z. Xiao-Lu, Gear drive design manual, Chemical industry press, pp. 594, 2010.

[7] L. Wen-Zhi, Finite element calculation and analysis on the column-shaped flex spline of harmonic drive, Journal of mechanical engineering, vol.42, pp. 52-57, 2006. 